AN AUDIENCE WITH...

\title{
Paul Stoffels
}

Paul Stoffels, a biopharma veteran who first joined Johnson \& Johnson (J\&J) in 2002 when it acquired Tibotec, stepped into his latest role within the company as Chief Scientific Officer (CSO) last year. Building on his broad experience leading the pharmaceutical research and development (RED) pipeline across all therapeutic areas - and in particular HIV, infectious diseases, central nervous system (CNS) and internal medicine RED he is now charged with setting the innovation agenda for JEJ's sprawling health-care empire. Partnering and a keen RED focus lie at the heart of his mission, he told Asher Mullard.

\section{Q How has your role changed since becoming CSO for J\&J in October?} I continue to have an important responsibility for the pharmaceutical group and I'm lucky to have a very good leader, Bill Hait, who takes responsibility of R\&D. And I continue to drive the sourcing of innovation: how do we get new products, how do we keep an open mind and how do we get the most out of open innovation?

But I now also have oversight of the innovation agenda across medical devices, diagnostics and the consumer sectors. I am looking at platforms that we can combine, such as biologics and devices, and drugs and devices, that will provide a lot of opportunities for us. We recently got a fibrin patch approved that provides an effective way to treat acute bleeding, but that had to be approved via the normal biologics license application route. Those types of developments would never happen if you don't work across different parts of a company.

It is the same with diagnostics. We have a lot of diagnostic developments now where there are significant cross-sector collaborations between the pharma and the diagnostic teams. More than 100 people today from our diagnostic groups are working on pharmaceutical development.

\section{Q] JUJ also launched four 'innovation} centres' to streamline its approach to partnering. How is this going? It's going very well. It was a big change for the company. Before launching these centres we would organize the deals we would do based on whether they were academic collaborations, venture capital collaborations, business development deals or otherwise. Now, we are going to several regions in the world - California, Boston, London and China - to focus first and foremost on the science and then we are being very flexible in how we collaborate. We want to bring all our capabilities, including capital, resourcing and expertise, and think about what each project needs. Every deal needs a different approach and we need to be very flexible in the way we are organized.

\section{Q Do you expect this restructuring to influence the number of deals you do?} You can't put metrics on deal making, because then you focus on how many deals you want to make. If you want 50 deals a year, you get 50 deals a year. Of course we are going to increase the number of deals, but it is all about how many valuable products we can bring to the organization.

But another aim of the innovation centres is to change the mindset internally on collaboration. We want our teams to focus not just on their science but more broadly on how we can improve our odds of success. Being out in regional hubs early increases our probability of success. If we know the products and the people better, we can make better decisions and we can get to the truth-telling experiments faster. If you don't stop a project, it will never stop. Having the most efficient way to get to those truth-telling experiments is important.

In Phase II, when a project has a shortcoming I always ask whether we could have known this earlier. I am quite often told that we knew the pharmacokinetics was 'a little this' and safety was 'a little that', but that we did the study anyway, and it failed late, so we lost US $\$ 20$ million. People by now have understood that it is not about being busy, it is about being successful. We have to create a culture in which we know how to fail earlier.

\section{Q How is your strategy of focusing your oncology R\&D on the tumour microenvironment working out?}

Focusing on the tumour microenvironment was a way for us to specialize and therefore get better and be more successful. It helps us to select and develop drugs. Abiraterone for metastatic castration-resistant prostate cancer, although not discovered internally, was a drug that came out of the approach. If you know an area very well, you can make more competitive bids to get the drugs you want. We can make high-risk decisions, and because we know what we know we have a high probability of success.

But focusing on one thing doesn't mean we don't do other things as well.

We are also focusing on disease areas, including haematological cancers, which is why we collaborated with Pharmacyclics on ibrutinib, and with Genmab for daratumumab.

\section{Q How has the recent failure of bapineuzumab impacted your CNS plans?}

It has encouraged us to go on. We had a positive biomarker effect, which shows that we can have some effect on the amyloid- $\beta$ disease pathway. But the trial was run in patients with very advanced disease; we may have treated our patients too late. Our scientists are now trying to figure out whether we would have been more successful if we had tested the same hypothesis earlier. 